\title{
ASSOCIATIVE REGULAR OPERATIONS ON GROUPS CORRESPONDING TO A PROPERTY $\odot$
}

\section{S. MORAN}

In previous papers $[4 ; 5 ; 6]$ we constructed and studied many classes of operations on groups which have the following properties:

(1) Associative;

(2) Commutative;

(3) The resultant group contains isomorphic images of the original factors and is generated by them;

(4) In the resultant group the intersection of a factor with normal subgroup generated by the remaining factors is the unit element.

Golovin [3] showed how to obtain all operations satisfying the last three conditions and called such operations regular multiplications.

Below we construct for every property $\odot$, applicable to groups, a corresponding regular multiplication. If $\odot$ persists homomorphically and ceases freely, we shall show that this multiplication is associative. $\rho$ is said to persist homomorphically if from the fact that a group satisfies $P$ it follows that every homomorphic image of the group also satisfies $P$. On the other hand, $\odot$ is said to cease freely if for every set of groups satisfying $P$ their free product does not satisfy $P$.

We introduce a number of preliminary concepts which are all contained in Moran [6]. $N(X)$ is said to be a normal subgroup function if it associates with every group $G$ a normal subgroup $N(G)$ of $G$. However, we are interested in the following more restrictive class of normal subgroup functions. $N(X)$ is said to be a strongly characteristic subgroup function if $N(X) \phi \leqq N(X \phi)$ for every homomorphism $\phi$ of $X$. This is equivalent to the condition that the normal subgroup be prescribed in such a manner that when extra defining relations are imposed on the group then the elements which originally belonged to the subgroup continue to remain therein. It seems that most of the well known normal subgroup functions, such as the Frattini subgroup, are of this type.

Let $F$ denote the free product of an arbitrary set of groups $G_{\alpha}$, $\alpha \in M$, and $N(X)$ be a normal subgroup function, then corresponding to $N(X)$ we have the normal subgroup $\left[N\left(G_{\alpha}\right), G_{\beta}\right]^{F}$ of $F$. $\left[N\left(G_{\alpha}\right), G_{\beta}\right]^{F}$ is the normal subgroup of $F$ generated by all commutators of the form $\left[g_{\alpha}, g_{\beta}\right]$, where $g_{\alpha} \in N\left(G_{\alpha}\right), g_{\beta} \in G_{\beta}$ and $\alpha$ and $\beta$ are distinct elements of the index set $M$. We now define the $N^{*}$-muitiplication of the groups $G_{\alpha}, \alpha \in M$, by ${ }^{1}$

Received by the editors December 23, 1958.

1 For further details the reader is referred to Moran [6]. 


$$
\prod_{\alpha \in M}^{N^{*}} G_{\alpha}=F /\left[N\left(G_{\alpha}\right), G_{\beta}\right]^{F}
$$

It follows from Golovin [3] that this defines a regular multiplication. In Moran [6] it was shown that the following conditions are sufficient to ensure the associativity of $N^{*}$-multiplication:

(a) $N(X)$ is a strongly characteristic subgroup function;

(b) $N(F)=1$ for all free products ${ }^{2} F$;

(c) $N\left(G_{\alpha}\right) \cdot\left[N\left(G_{\alpha}\right), G_{\beta}\right]^{F} /\left[N\left(G_{\alpha}\right), G_{\beta}\right]^{F} \leqq N\left(\prod_{\alpha \in M}^{N^{*}} G_{\alpha}\right)$ for every $\alpha$ in $M$.

We now construct for every property $\beta$ the following normal subgroup function $P(X)$ :

$P(X)$ is the union of all subgroups satisfying the property $\odot$ which are normal in $X$. If $X$ has no such subgroup, then $P(X)$ is taken to be the trivial subgroup.

LeMma 1. If $\odot$ persists homomorphically, then $P(X)$ is a strongly characteristic subgroup function.

Proof. Let $H$ be a normal subgroup of $X$ and suppose $H$ satisfies $P$. If $\phi$ is a homomorphism of $X$, then $H \phi$ satisfies $\beta$ and is normal in $X \phi$. Hence $H \phi$ is contained in $P(X \phi)$, giving the required result.

Lemma 2. If $\odot$ persists homomorphically and ceases freely, then a free product has no nontrivial normal subgroups satisfying $P$.

Proof. Suppose that the free product of the groups $H_{\beta}, \beta \in N$, satisfies the property $P$. As $P$ persists homomorphically it follows that all the free factors $H_{\beta}$ also must satisfy $\odot$. However, this contradicts the fact that $P$ ceases freely. Hence a free product does not satisfy $P$. Now by the Kuroš subgroup theorem [7], a normal subgroup of a free product is either a free product or the infinite cyclic group or the infinite dihedral group. The first possibility is ruled out by the above argument. It was proved in Moran [6] that a free product does not have nontrivial normal abelian subgroups. Lastly as the infinite dihedral group has a fully invariant cyclic subgroup, a free product does not have the infinite dihedral group as a normal subgroup. Hence the required result follows.

We are now in a position to state the following main result concerning the $P^{*}$-multiplication which is defined by

2 Throughout this paper free product denotes a free product which has more than one free factor. We also exclude the infinite dihedral group. Because of the latter restriction, the associativity of the $N^{*}$-multiplication may fail in the one particular case when $G_{\alpha_{1}}$ and $G_{\alpha_{2}}$ are cyclic groups of order two, namely, $\left(G_{\alpha_{1}} N^{*} G_{\alpha_{2}}\right) N^{*}\left(\prod_{\alpha \neq \alpha_{1}, \alpha_{2}}^{N^{*}} G_{\alpha}\right)$ not $\cong \prod_{\alpha \in M}^{N^{*}} G_{\alpha}$. 


$$
\prod_{\alpha \in M}^{P^{*}} G_{\alpha}=F /\left[P\left(G_{\alpha}\right), G_{\beta}\right]^{F}
$$

THEOREM. If $P$ persists homomorphically and ceases freely, then the corresponding $P^{*}$-multiplication is an associative regular multiplication.

Proof. The above conditions (a) and (b) for associativity follow from Lemmas 1 and 2. It remains to show that

$$
P\left(G_{\alpha}\right) \cdot\left[P\left(G_{\alpha}\right), G_{\beta}\right]^{F} /\left[P\left(G_{\alpha}\right), G_{\beta}\right]^{F} \leqq P\left(\prod_{\alpha \in M} P^{*} G_{\alpha}\right)
$$

for every $\alpha$ in $M$. Let $H_{\alpha}$ be a normal subgroup of $G_{\alpha}$ and suppose further that $H_{\alpha}$ satisfies $\rho$, then $H_{\alpha}$ is one of the generating subgroups of $P\left(G_{\alpha}\right)$. It is obviously true that $H_{\alpha} \cdot\left[P\left(G_{\alpha}\right), G_{\beta}\right]^{F} /\left[P\left(G_{\alpha}\right), G_{\beta}\right]^{F}$ satisfies $P$ and is normal in $\prod^{P^{*}} G_{\alpha}$.

It is interesting to notice that the value of $P(X)$ for the associative $P^{*}$-multiplication is given by

$$
P\left(\prod_{\alpha \in M}^{P^{*}} G_{\alpha}\right)=\prod_{\alpha \in M}{ }^{x \Rightarrow x} P\left(G_{\alpha}\right) .
$$

Examples of properties $\rho$ of the above type are given by nilpotency, solubility, finiteness and periodicity. Further examples are obtained by imposing any one of the first three conditions locally. Another interesting example arises when $\rho$ denotes simplicity.

Corresponding to a property $\mathcal{P}$, there are many ways of obtaining a subgroup function $P(X)$. The following would seem to be of particular interest as all the above results hold also for them after suitable changes in terminology:

$P(X)$ is the union of all accessible subgroups of $X$ satisfying $^{3} \odot$;

$P(X)$ is the union of all minimal normal subgroups of $X$ satisfying $P$;

$P(X)$ is the union of all minimal accessible subgroups of $X$ satisfying $^{4} P$.

The other cases that suggest themselves are obtained on replacing union by intersection and minimal by maximal with the added possible restriction that subgroups must be of finite index. ${ }^{5}$ However, the corresponding question concerning the associativity of their $P^{*}$ multiplications is a much more difficult one and has not been settled

3 This subgroup function has been studied by R. Baer [2] in the particular case when $P$ denotes nilpotency.

- $P$ can be taken to be vacuous.

${ }^{5}$ See for instance the Baer-Frattini subgroup [1]. 
even in the case when $P$ is vacuous. In this connection it is of interest to observe that when $\rho$ denotes nilpotency or local nilpotency some of the subgroup functions that arise are the same as those given above.

\section{REFERENCES}

1. R. Baer, Das Hyperzentrum einer Gruppe III, Math. Z. vol. 59 (1953) pp. 299338.

2. - Nilgruppen, Math. Z. vol. 62 (1955) pp. 402-437.

3. O. N. Golovin, Nilpotent products of groups, Mat. Sb. vol. 27 (69) (1950) pp. $427-454$.

4. S. Moran, Associative operations on groups I, Proc. London Math. Soc. (3) vol. 6 (1956) pp. 581-596.

5. - Associative operations on groups II, Proc. London Math. Soc. (3) vol. 8 (1958) pp. 548-568.

6. - Associative operations on groups III, Proc. London Math. Soc. (3) vol. 9 (1959) pp. 287-317.

7. M. Takahasi, Bemerkungen über den Untergruppensatz in Freien Produkte, Proc. Imp. Akad. Tokyo vol. 20 (1944) pp. 589-594.

The University of Glasgow, Glasgow, Scotland 